\title{
Congruences on Dynamic Algebras
}

\author{
SANDRA MARQUES PINTO, Departamento de Matemática, Universidade \\ de Coimbra, Portugal. \\ E-mail:sandra@mat.uc.pt
}

TERESA OLIVEIRA-MARTINS, Departamento de Matemática, Universidade de Coimbra, Portugal.

E-mail:meresa@mat.uc.pt

M. CÉU PINTO, Departamento de Matemática, Universidade de Coimbra, Portugal.

E-mail:pinto@mat.uc.pt

\begin{abstract}
The lattice $\operatorname{Cong} \mathcal{D}$ of all dynamic congruences on a given dynamic algebra $\mathcal{D}$ is presented. Whenever $\mathcal{D}$ is separable with zero we define dynamic ideal on $\mathcal{D}$, given rise to the lattice $I d e \mathcal{D}$. The notions of kernel of a dynamic congruence and the congruence generated by a dynamic ideal are introduced to describe a Galois connection between $\operatorname{Cong} \mathcal{D}$ and $I d e \mathcal{D}$. We study conditions under which a dynamic congruence is determined by its kernel.

Keywords: Boolean algebra with operators, dynamic algebra, dynamic congruence, dynamic ideal, Galois connection.
\end{abstract}

\section{Introduction}

To enhance the importance of the algebraic structure of the regular part of a dynamic algebra, we introduce the notions of dynamic congruence and dynamic ideal on dynamic algebras viewed as heterogeneous algebras, differing from those presented in dynamic algebras regarded as modal algebras, [6].

\section{Dynamic algebras}

Dynamic algebras were introduced by Kozen [7] and Pratt [9] to provide models for Propositional Dynamic Logic $(P D L)$. Following Pratt a dynamic algebra is a two-sorted algebra $(\mathcal{B}, \mathcal{R},\langle\rangle)$ verifying a certain set of equations. The Boolean universe $\mathcal{B}=(B, \vee, \sim, 0)$ is a Boolean algebra (an algebra with a binary operation $\vee$, one unary operation $\sim$ and a nullary operation 0 , satisfying the usual set of axioms); the regular universe $\mathcal{R}=\left(R, \cup, ;,{ }^{*}\right)$ (or universe of actions or universe of programs) is an algebra with two binary operations, $\cup$,; and one unary operation ${ }^{*}$. The heterogeneous operator (diamond) $\langle\rangle:, R \times B \longrightarrow B$ relates both algebras.

DeFinition 2.1

A dynamic algebra $\mathcal{D}=\left(\mathcal{B}=(B, \vee, \sim, 0), \mathcal{R}=\left(R, \cup, ;,{ }^{*}\right),\langle\rangle,\right)$ is an algebra satisfying

(C) The Author, 2007. Published by Oxford University Press. All rights reserved. For Permissions, please email: journals.permissions@oxfordjournals.org doi:10.1093/jigpal/jzm014 


\section{Congruences on Dynamic Algebras}

(1) $\mathcal{B}$ is a Boolean algebra

(2a) $\langle a, 0\rangle=0$

(2b) $\langle a, p \vee q\rangle=\langle a, p\rangle \vee\langle a, q\rangle$

(3) $\langle a \cup b, p\rangle=\langle a, p\rangle \vee\langle b, p\rangle$

(4) $\langle a ; b, p\rangle=\langle a,\langle b, p\rangle\rangle$

(5a) $p \vee\left\langle a ; a^{*}, p\right\rangle \leq\left\langle a^{*}, p\right\rangle$

(5b) $\left\langle a^{*}, p\right\rangle \leq p \vee\left\langle a^{*}, \sim p \wedge\langle a, p\rangle\right\rangle$

for $p, q \in B$ and $a, b \in R$.

\section{Notes.}

1. For $p$ and $q$ elements of the Boolean algebra $\mathcal{B}$, we used $(p \leq q)$ as an abbreviation of $(p \vee q$ $=q)$.

2. Pratt [9] showed that conditions (5a) and (5b) can be replaced by $\left\langle a^{*}, p\right\rangle=\min a ! p$ where $a ! p=\{q \in B: p \vee a q \leq q\}$.

Notation. For $a, b \in R$ and $p \in B$ we write $a p$ instead of $\langle a, p\rangle, a b$ instead of $a ; b$ and use $a \leq b$ whenever $(a p \leq b p$, for every $p \in B$ ).

Kripke structures, the traditional models for $P D L$ [4], were presented in [9] as examples of dynamic algebras. They are defined as follows. The full Kripke structure on a given nonempty set $S$ is a triple $(S, \mathcal{P}(S), \mathcal{P}(S \times S))$ where $\mathcal{P}(S)$ is the Boolean algebra of all subsets of $S$ (with the usual set theoretical operations) and $\mathcal{P}(S \times S)$ is the set of all binary relations on $S$. The operations ; $\cup$ and ${ }^{*}$ are, respectively, the composition, the union and the reflexive transitive closure of binary relations. The diamond operation $\langle a, p\rangle$ (for $a \in \mathcal{P}(\mathcal{S} \times \mathcal{S}$ ) and $p \in \mathcal{P}(\mathcal{S})$ ) is defined to be the pre-image of $p$ under $a$,

$$
\left\{s \in S:\left(s, s^{\prime}\right) \in a \text {, for some } s^{\prime} \in p\right\} .
$$

Kripke structures are defined as the dynamic subalgebras of full Kripke structures. We denote by $D A$ the variety of all dynamic algebras. Pratt [9] proved that

$$
D A=H S P(K r i \cup T)
$$

where Kri denotes the class of all Kripke structures and $T$ is the class of all dynamic algebras with trivial Boolean part.

Let $\mathcal{D}=(\mathcal{B}, \mathcal{R},\langle\rangle) \in D A$ and $a, b \in R$. We put $a \cong_{\mathcal{R}} b$, if $a p=b p$, for every proposition $p$ in $B$. The pair $\left(=, \cong_{\mathcal{R}}\right.$ ) defines a congruence relation ( $c f$. Def.2.3, below) in the dynamic algebra $\mathcal{D}$. A dynamic algebra is separable if for any actions $a$ and $b$ in $R$, such that $a p=b p$, for any proposition $p \in B$, then $a=b$. For every dynamic algebra $\mathcal{D}, \mathcal{D} /\left(=, \cong_{\mathcal{R}}\right)$ is separable. We use $S D A$ to denote the class of all separable dynamic algebras.

The mixed operator diamond $\langle\rangle:, R \times B \longrightarrow B$ can be seen as

$$
\begin{aligned}
\langle\rangle: R & \longrightarrow F(B ; B) \\
a & \longrightarrow(p \longrightarrow\langle a\rangle p=\langle a, p\rangle)
\end{aligned}
$$

where $F(B ; B)=\{f \mid f: B \longrightarrow B$ is a mapping $\}$. However, unless $\mathcal{D}$ is separable, \langle\rangle does not define a representation of $R$ since \langle\rangle is not one-to-one. We may have equal mappings $\langle a\rangle$ and $\langle b\rangle$ in $F(B ; B)$ with $a \neq b$. Consequently, for $\mathcal{D}$ an arbitrary dynamic algebra, $\{\langle a\rangle: a \in R\}$ is a family of mappings in $B$ indexed by $R$ (where the extensionality axiom is not satisfied). In a separable dynamic algebra, $R$ may be identified with a set of mappings in $B$, that we will use whenever appropriate. Therefore, a dynamic algebra $\mathcal{D}$, can be viewed as a Boolean algebra 
with operators [9]. Moreover, defining, for each $a \in R,[a] p:=\sim\langle a, \sim p\rangle$ for every $p \in B$, then $\left(\mathcal{B},\{[a]\}_{a \in R}\right)$ is a modal algebra $(c f .[6])$ associated with $\mathcal{D}$. The same modal algebra may be determined by distinct dynamic algebras.

The relation $\leq$ in $R, a \leq b$ whenever $(a p \leq b p$, for every $p \in B$ ) is reflexive and transitive, but it is not antisymmetric in general (since the conditions $a p \leq b p$ and $b p \leq a p$ give rise to $a p=b p$, for every $p \in B$ and not necessarily to $a=b$ ) and, therefore, defines a quasi-order in $R$. Clearly, in separable dynamic algebras $\leq$ defines a partial order since the antisymmetry is satisfied.

The elementary algebraic theory of dynamic algebras is similar to that of every other algebraic system [10], [11]. We will specify some concepts for later use.

Definition 2.2

Let

$$
\begin{aligned}
& \mathcal{D}=(\mathcal{B}=(B, \vee, \sim, 0), \mathcal{R}=(R, \cup, ;, *),\langle\rangle) \text { and } \\
& \mathcal{D}^{\prime}=\left(\mathcal{B}^{\prime}=\left(B^{\prime}, \vee^{\prime}, \sim^{\prime}, 0^{\prime}\right), \mathcal{R}^{\prime}=\left(R^{\prime}, \cup^{\prime}, ;^{\prime}, *^{\prime}\right),\langle\rangle^{\prime}\right)
\end{aligned}
$$

be dynamic algebras and $h_{1}: B \longrightarrow B^{\prime}$ and $h_{2}: R \longrightarrow R^{\prime}$ be mappings. The pair $h=\left(h_{1}, h_{2}\right)$ is a dynamic homomorphism between the dynamic algebras $\mathcal{D}$ and $\mathcal{D}^{\prime}$ if $h_{1}$ and $h_{2}$ preserve the mentioned one-sorted operations and $h$ preserves the mixed operation, i.e., for $p \in B, a, b \in R$ we have

1. $h_{1}$ is a Boolean homomorphism

2. $h_{2}(a \cup b)=h_{2}(a) \cup^{\prime} h_{2}(b)$

3. $h_{2}(a ; b)=h_{2}(a) ;{ }^{\prime} h_{2}(b)$

4. $h_{2}\left(a^{*}\right)=\left(h_{2}(a)\right)^{* \prime}$

5. $h_{1}(\langle a\rangle p)=\left\langle h_{2}(a)\right\rangle^{\prime} h_{1}(p)$.

DeFinition 2.3

Let $\mathcal{D}=(\mathcal{B}, \mathcal{R},\langle\rangle)$ be a dynamic algebra. The pair $\theta=\left(\theta_{1}, \theta_{2}\right)$ is a (dynamic) congruence relation on $\mathcal{D}$ if $\theta_{1}$ is a congruence relation on $\mathcal{B}, \theta_{2}$ is a congruence relation on $\mathcal{R}$ and ap $\theta_{1} b q$ whenever $\left(p \theta_{1} q\right.$ and $\left.a \theta_{2} b\right)$.

In a dynamic algebra $\mathcal{D}=(\mathcal{B}, \mathcal{R},\langle\rangle)$ we define the congruences $\Delta_{B}$ and $\nabla_{B}$ on $B$ and $\Delta_{R}$ and $\nabla_{R}$ on $R$ as expected:

$$
\begin{array}{ll}
\Delta_{B}=\{(p, p): p \in B\} & \nabla_{B}=\{(p, q): p, q \in B\} \\
\Delta_{R}=\{(a, a): a \in R\} & \nabla_{R}=\{(a, b): a, b \in R\} .
\end{array}
$$

One can easily show that the pairs $\left(\Delta_{B}, \Delta_{R}\right),\left(\nabla_{B}, \nabla_{R}\right)$ and $\left(\nabla_{B}, \Delta_{R}\right)$ are congruences on $\mathcal{D}$, but, in general, $\left(\Delta_{B}, \nabla_{R}\right)$ is not a congruence on $\mathcal{D}$.

As usual, we define $\mathcal{B} / \theta_{\theta_{1}}=\left\{[p]_{\theta_{1}}: p \in B\right\}$ and $\mathcal{R} / \theta_{\theta_{2}}=\left\{[a]_{\theta_{2}}: a \in R\right\}$. The structure $\mathcal{D} /{ }_{\theta}=$ $\left(\mathcal{B} / \theta_{1}, \mathcal{R} / \theta_{\theta_{2}},\langle\rangle\right)$ is a dynamic algebra (the quotient dynamic algebra) with operations defined by

$$
\begin{aligned}
{[p]_{\theta_{1}} \vee[q]_{\theta_{1}}:=[p \vee q]_{\theta_{1}} } & & {[a]_{\theta_{2}} \cup[b]_{\theta_{2}}:=[a \cup b]_{\theta_{2}} } \\
\sim[p]_{\theta_{1}}:=[\sim p]_{\theta_{1}} & & {[a]_{\theta_{2}} ;[b]_{\theta_{2}}:=[a ; b]_{\theta_{2}} } \\
{[a]_{\theta_{2}}[p]_{\theta_{1}}:=[a p]_{\theta_{1}} } & \text { and } & {[a]_{\theta_{2}}^{*}:=\left[a^{*}\right]_{\theta_{2}} . }
\end{aligned}
$$

The algebra $\left(\mathcal{B} / \theta_{1}, \mathcal{R} / \theta_{2},\langle\rangle\right)$ is the homomorphic image of $(\mathcal{B}, \mathcal{R},\langle\rangle)$ under the natural homomorphism $\nu=\left(v_{1}, v_{2}\right)$ defined by

$\nu_{1}: p \mapsto[p]_{\theta_{1}}$

$\nu_{2}: a \mapsto[a]_{\theta_{2}}, p \in B, a \in R$. 


\section{Congruences on Dynamic Algebras}

Also, associated with each homomorphism, there exists a natural congruence. If $h: \mathcal{D} \longrightarrow \mathcal{D}^{\prime}$ is a dynamic algebra homomorphism, the kernel relation $\operatorname{ker}_{h}=\left(K_{1}, K_{2}\right)$ given by

$$
\begin{aligned}
& p K_{1} q \text { if and only if } h_{1}(p)=h_{1}(q) \\
& a K_{2} b \text { if and only if } h_{2}(a)=h_{2}(b)
\end{aligned}
$$

for every $p, q \in B$ and $a, b \in R$, defines a congruence on $\mathcal{D}$.

The homomorphism theorem enable us to assert that every homomorphic image of a dynamic algebra $\mathcal{D}$ is, up to an isomorphism, a quotient algebra [11].

Theorem 2.4

If $\mathcal{D}=(\mathcal{B}, \mathcal{R},\langle\rangle)$ and $\mathcal{D}^{\prime}$ are dynamic algebras with $\mathcal{D}^{\prime}$ homomorphic image of $\mathcal{D}$ under $h: \mathcal{D} \longrightarrow \mathcal{D}^{\prime}$, then $\mathcal{D}^{\prime}$ is isomorphic to $\mathcal{D} /$ ker $_{h}$ under the isomorphism $\psi: \mathcal{D} /$ ker $_{h} \longrightarrow \mathcal{D}^{\prime}$ defined by $\psi_{1}[p]_{K_{1}}:=h_{1}(p)$ and $\psi_{2}[a]_{K_{2}}:=h_{2}(a), p \in B, a \in R$.

\section{The lattice $\operatorname{CongD}$}

Denote by $\operatorname{Cong} \mathcal{D}$ the set of all dynamic congruences defined on a dynamic algebra $\mathcal{D}$. This set is partially ordered by $\left(\theta_{1}, \theta_{2}\right) \leq\left(\gamma_{1}, \gamma_{2}\right)$ if and only if $\theta_{1} \subseteq \gamma_{1}$ and $\theta_{2} \subseteq \gamma_{2}$. Our next aim is to define the lattice $\left(\operatorname{Cong} \mathcal{D}, \wedge_{\mathcal{D}}, \vee_{\mathcal{D}}\right)$. Since the intersection, $\theta \cap \gamma=\left(\theta_{1} \cap \gamma_{1}, \theta_{2} \cap \gamma_{2}\right)$, of any two dynamic congruences $\theta$ and $\gamma$ defined on $\mathcal{D}$ is, itself, a dynamic congruence on $\mathcal{D}$, let $\theta \wedge_{\mathcal{D}} \gamma:=\theta \cap \gamma$. Let us use $\langle\theta\rangle_{\mathcal{A}}$ to represent the congruence relation generated by the binary relation $\theta$ on any (homogeneous or heterogeneous) algebra $\mathcal{A}$, i.e., the intersection of all congruence relations $\theta^{\prime}$ on $\mathcal{A}$ containing $\theta$,

$$
\langle\theta\rangle_{\mathcal{A}}:=\cap\left\{\theta^{\prime}: \theta^{\prime} \in \operatorname{Cong} \mathcal{A} \text { and } \theta \subseteq \theta^{\prime}\right\}
$$

Now we need to define $\theta \vee_{\mathcal{D}} \gamma=\left(\tau_{1}, \tau_{2}\right)$. It is immediate that the regular part of the congruence $\theta \vee_{\mathcal{D}} \gamma$ can be given by $\tau_{2}:=\theta_{2} \vee_{\mathcal{R}} \gamma_{2}=\left\langle\theta_{2} \cup \gamma_{2}\right\rangle_{\mathcal{R}}$, using the classic definition of supremum of two congruences. As far as the Boolean part, some caution is required. Since the Boolean part must be closed to the diamond operation evolving elements of $R$, we could be led to think of enlarging $\left\langle\theta_{1} \cup \gamma_{1}\right\rangle_{\mathcal{B}}$ with, for instance, elements of the type $(a p, b q)$ with $(a, b) \in \theta_{2}$ and $(p, q) \in \gamma_{1}$. In fact, that is not necessary, as shown below.

Proposition 3.1

Let $\mathcal{D}$ be a dynamic algebra. For $\theta, \gamma \in \operatorname{Cong} \mathcal{D},(p, q) \in\left\langle\theta_{1} \cup \gamma_{1}\right\rangle_{\mathcal{B}}$ and $(a, b) \in\left\langle\theta_{2} \cup \gamma_{2}\right\rangle_{\mathcal{R}}$ we have $(a p, b q) \in\left\langle\theta_{1} \cup \gamma_{1}\right\rangle_{\mathcal{B}}$.

Proof. Let $(p, q) \in\left\langle\theta_{1} \cup \gamma_{1}\right\rangle_{\mathcal{B}}$ and $(a, b) \in\left\langle\theta_{2} \cup \gamma_{2}\right\rangle_{\mathcal{R}}$. By [10], we know that there exist natural numbers $n, m$, a sequence $p_{1}, p_{2}, p_{3}, \ldots, p_{n}$ in $B$ and a sequence $a_{1}, a_{2}, a_{3}, \ldots, a_{m}$ in $R$ such that

$$
\begin{array}{llll}
p \theta_{1} p_{2}, & p_{2} \gamma_{1} p_{3}, & p_{3} \theta_{1} p_{4}, \cdots, & p_{n-1} \gamma_{1} q \\
a \theta_{2} a_{2}, & a_{2} \gamma_{2} a_{3}, & a_{3} \theta_{2} a_{4}, \cdots, & a_{m-1} \gamma_{2} b .
\end{array}
$$


Since we always can repeat elements in the sequences, we are allowed to put $m=n$. So,

$$
{ }_{a p \theta_{1}} a_{2} p_{2}, a_{2} p_{2} \gamma_{1} a_{3} p_{3}, a_{3} p_{3} \theta_{1} a_{4} p_{4}, \cdots, a_{n-1} p_{n-1} \gamma_{1} b q \text {. }
$$

But this means that $(a p, b q) \in\left\langle\theta_{1} \cup \gamma_{1}\right\rangle_{\mathcal{B}}$, as required.

The structure $\left(\operatorname{Cong} \mathcal{D}, \wedge_{\mathcal{D}}, \vee_{\mathcal{D}}\right)$ where, for every $\theta, \gamma \in \operatorname{Cong} \mathcal{D}$, the operations are defined by

$$
\begin{aligned}
& \theta \wedge_{\mathcal{D}} \gamma=\theta \cap \gamma=\left(\theta_{1} \cap \gamma_{1}, \theta_{2} \cap \gamma_{2}\right) \\
& \theta \vee_{\mathcal{D}} \gamma=\langle\theta \cup \gamma\rangle_{\mathcal{D}}=\left(\left\langle\theta_{1} \cup \gamma_{1}\right\rangle_{\mathcal{B}},\left\langle\theta_{2} \cup \gamma_{2}\right\rangle_{\mathcal{R}}\right)
\end{aligned}
$$

is a lattice, called the congruence lattice $\operatorname{Cong} \mathcal{D}$ of $\mathcal{D}$. Elements $\theta=\left(\theta_{1}, \theta_{2}\right)$ and $\gamma=\left(\gamma_{1}, \gamma_{2}\right)$ of Cong $\mathcal{D}$ are said to be permutable or $\theta$ and $\gamma$ permute if and only if $\theta \circ \gamma=\gamma \circ \theta$, i.e., $\theta_{1} \circ \gamma_{1}=\gamma_{1} \circ \theta_{1}$ and $\theta_{2} \circ \gamma_{2}=\gamma_{2} \circ \theta_{2}$.

A class $K$ of (homogeneous or heterogeneous) algebras is congruence-permutable if every algebra $A$ on $K$ is congruence-permutable, i.e., if every pair of congruences on $A$ permutes. Although congruences on Boolean algebras are permutable, it can be easily proved that the variety generated by the class of all separable dynamic algebras, $V(S D A)$, is not congruencepermutable.

\section{Proposition 3.2}

The variety $V(S D A)$ is a non congruence-permutable class.

Proof. Let $\mathcal{D}=(\mathcal{P}(S),\{a, b, e\},\langle\rangle)$, for $S=\{1,2\}, a=\{(1,1),(2,2)\}, b=\{(2,2)\}$ and $e=\emptyset$. For the dynamic congruences $\alpha=\left(\alpha_{1}, \alpha_{2}\right)$ and $\beta=\left(\beta_{1}, \beta_{2}\right)$ with $\alpha_{1}=\mathcal{P}(S) \times \mathcal{P}(S)$, $\alpha_{2}=\{(a, a),(b, b),(e, e),(a, b),(b, a)\}, \beta_{1}=\mathcal{P}(S) \times \mathcal{P}(S)$ and $\beta_{2}=\{(a, a),(b, b),(e, e),(b, e),(e, b)\}$ we have $(a, e) \in \alpha_{2} \circ \beta_{2}$ but $(a, e) \notin \beta_{2} \circ \alpha_{2}$.

As in the homogeneous case, the supremum of permutable dynamic congruences is given by their composition.

A class $K$ of (homogeneous or heterogeneous) algebras is congruence-distributive if, for every algebra $\mathcal{A}$ on $K$, the lattice $\operatorname{Cong} \mathcal{A}$ is distributive.

\section{Proposition 3.3}

The variety $D A$ is a non distributive-congruence class.

Proof. Let $\mathcal{D}=(\mathcal{B}, \mathcal{R},\langle\rangle)$ be the dynamic algebra, where $\mathcal{B}$ is the trivial Boolean algebra $B=\{0\}, \mathcal{R}$ is defined (cf. [5, Fig.3]) by $R=\{a, b, c, d$, o $\}$ with

$$
\begin{aligned}
& a \cup a=a \quad b \cup b=b \quad c \cup c=c \quad d \cup d=d \quad \mathrm{o}=\mathrm{o} \\
& a \cup b=c \quad b \cup c=c \quad c \cup d=d \quad d \cup \mathrm{o}=d \\
& a \cup c=c \quad b \cup d=d \quad c \cup \mathrm{o}=c \\
& a \cup d=d \quad b \cup \mathrm{o}=b \\
& a \cup \mathrm{o}=a \\
& m ; n=\mathrm{o} \\
& d ; l=l ; d=l \\
& l^{*}=l \text {, }
\end{aligned}
$$

and, obviously, $\langle l, 0\rangle=0$, for every $l \in R$ and $m, n \in\{a, b, c, o\}$. 


\section{Congruences on Dynamic Algebras}

Let $\alpha=\left(\alpha_{1}, \alpha_{2}\right), \beta=\left(\beta_{1}, \beta_{2}\right)$ and $\gamma=\left(\gamma_{1}, \gamma_{2}\right)$ be dynamic congruences defined by $\alpha_{1}=\beta_{1}=\gamma_{1}=\{(0,0)\}=\Delta_{B}$

$\alpha_{2}=\{(a, a),(b, b),(c, c),(d, d),(\mathrm{o}, \mathrm{o}),(b, c),(c, b)\}$

$\beta_{2}=\{(a, a),(b, b),(c, c),(d, d),(\mathrm{o}, \mathrm{o}),(a, \mathrm{o}),(\mathrm{o}, a),(b, c),(c, b)\}$

$\gamma_{2}=\{(a, a),(b, b),(c, c),(d, d),(\mathrm{o}, \mathrm{o}),(a, c),(c, a),(b, \mathrm{o}),(\mathrm{o}, b)\}$

We have $\alpha \vee(\beta \wedge \gamma)=\alpha$ and $(\alpha \vee \beta) \wedge(\alpha \vee \gamma)=\beta$. So $D A$ is a non distributive-congruence variety.

A variety $V$ of (homogeneous or heterogeneous) algebras is arithmetical if, each $A$ in $V$ is congruence-permutable and congruence-distributive. It is well known, (cf. [12]), that for any class $K$ of homogeneous algebras with a common discriminator term, $V(K)$ is arithmetical. Adaptations of this results can be established for any class $K$ of dynamic algebras.

DeFinition 3.4

If $\mathcal{D}=(\mathcal{B}, \mathcal{R},\langle\rangle)$ is a dynamic algebra, a pair of terms on the language of $\mathcal{D},\left(t_{1}(x, y, z)\right.$, $\left.t_{2}\left(x^{\prime}, y^{\prime}, z^{\prime}\right)\right)$, is said to be a pair of discriminator terms whenever, for all $p, q, r \in B$ and $a, b, c \in R$

$$
\begin{aligned}
& t_{1}(p, q, r)= \begin{cases}r & \text { if } p=q \\
p & \text { if } p \neq q\end{cases} \\
& t_{2}(a, b, c)= \begin{cases}c & \text { if } a=b \\
a & \text { if } a \neq b,\end{cases}
\end{aligned}
$$

i.e., $t_{1}(x, y, z)$ is a discriminator term on $\mathcal{B}$ and $t_{2}\left(x^{\prime}, y^{\prime}, z^{\prime}\right)$ is a discriminator term on $\mathcal{R}$.

LEMMA 3.5

Let $K$ be a class of dynamic algebras with a common pair of discriminator terms. Then $V(K)$ is arithmetical.

Proposition 3.6

Let $K$ be the class of the simple algebras of $S D A$. Then $V(K)$ is a proper subvariety of $V(S D A)$ (and, consequently of $D A$ ).

Proof. Since simple separable dynamic algebras are of the type $\left(B,\left\{\exists_{s}\right\},\langle\rangle\right)$, for $B$ arbitrary Boolean algebra with the singular element $\exists_{s}$ of the regular part defined by $\exists_{s}(p)=1$, for all $\mathrm{p} \neq 0, \mathrm{p} \in B$ and $\exists_{s}(0)=0,[8]$, the pair of terms $\left(t_{1}, t_{2}\right)$ given by

$$
\begin{aligned}
t_{1}(x, y, z) & =\left(\exists_{s}(x+y) \wedge x\right) \vee\left(\sim \exists_{s}(x+y) \wedge z\right) \\
t_{2}\left(x^{\prime}, y^{\prime}, z^{\prime}\right) & =x^{\prime}
\end{aligned}
$$

where the symbol + denotes the symmetric Boolean difference operation, establishes a pair of discriminator terms on the class $K\left(c f\right.$. [12] for $\left.t_{1}\right)$. Therefore $V(K)$ is an arithmetical variety. But $D A$ and $V(S D A)$ are non congruence-permutable varieties.

\section{Separating congruence}

Let $\mathcal{D}=(\mathcal{B}, \mathcal{R},\langle\rangle)$ be a dynamic algebra. In this paragraph we are going to show that, corresponding to a dynamic congruence $\theta=\left(\theta_{1}, \theta_{2}\right)$ on $\mathcal{D}$, there exists always a dynamic 
congruence $\phi$ on $\mathcal{D}$ with the same Boolean part such that $\mathcal{D} / \phi$ is separable ( $c f$. Prop.4.2). We also verify that, in general, $\theta_{1}$ does not determine $\theta_{2}$. For example, if $\mathcal{D} / \theta$ is not separable and $\gamma=\left(\theta_{1}, \gamma_{2}\right)$ is given by $a \gamma_{2} b$ if and only if $a p \theta_{1} b p$, for all $p \in B$, then $\gamma$ is a congruence on $\mathcal{D}$, distinct from $\theta$ ( $c f$. Prop.4.2). That is the case of Ex.5.13.

There are Boolean congruences on $\mathcal{D}$ that are not the Boolean part of any of its dynamic congruences. Let $\mathcal{B}$ the Boolean algebra with four elements $\{0, p, q, 1\}$ and $R=\{\mathrm{a}\}$ where $a$ is defined by $a 0=0$ and $a p=a q=a 1=1$. Since $I_{1}=\{0, p\}$ is a Boolean ideal on $\mathcal{B}$, we can affirm that $\theta_{1}$ defined by, for $s, r \in B,(s, r) \in \theta_{1}$ iff $s \vee i=r \vee i$, for some $i \in I_{1}$, is a Boolean congruence on $\mathcal{D}=(\mathcal{B}, \mathcal{R},\langle\rangle)$ with congruence classes $[0]_{\theta_{1}}=\{0, p\}$ and $[q]_{\theta_{1}}=\{q, 1\}$. But Cong $\mathcal{R}$ is the singular set $\left\{\Delta_{R}=\{(a, a)\}\right\}$ and $\left(\theta_{1}, \Delta_{R}\right)$ is not a dynamic congruence on $\mathcal{D}$, since $(0, p) \in \theta_{1}$ and $(a, a) \in \Delta_{\mathrm{R}}$ but $(a 0, a p) \notin \theta_{1}$. Therefore, the dynamic algebra $\mathcal{D}$ has a Boolean congruence $\theta_{1}$ which is not the Boolean part of any of its dynamic congruences.

A Boolean congruence $\theta_{1}$ is called dynamical on $\mathcal{D}=(\mathcal{B}, \mathcal{R},\langle\rangle)$ whenever there exists a congruence $\theta_{2}$ on $\mathcal{R}$ such that $\left(\theta_{1}, \theta_{2}\right)$ is a congruence on $\mathcal{D}$.

A dynamic algebra $\mathcal{D}=(\mathcal{B}, \mathcal{R},\langle\rangle)$ determines, as previously seen, a modal algebra $(\mathcal{B},\{[a]: a \in R\})$. In any modal algebra, $\left(\mathcal{B},\left\{f_{i}\right\}_{i<k}\right)$ (where $k$ denotes an arbitrary ordinal), there exists an isomorphism between the lattice of the open filters (filters $F$ on $\mathcal{B}$ satisfying condition: if $p \in F$ then $f_{i} p \in F$, for every $i<k$ ), and the lattice of the modal congruences, $(c f$. [6 Thm.4.1.10]). In this context since a dynamical congruence is a modal congruence, we can affirm that to each dynamical congruence corresponds a unique open filter on $\mathcal{B}$.

Proposition 4.1

Let $\theta_{1}$ be a Boolean congruence on $\mathcal{D}$. Then $\theta_{1}$ is dynamical on $\mathcal{D}$ if and only if $\left(\theta_{1}, \Delta_{\mathcal{R}}\right)$ is a dynamic congruence on $\mathcal{D}$.

Proposition 4.2

Let $\theta_{1}$ be a dynamical congruence on $\mathcal{D}=(\mathcal{B}, \mathcal{R},\langle\rangle)$ and let $\phi=\left(\phi_{1}, \phi_{2}\right)$ be defined by

$$
\begin{aligned}
\phi_{1} & =\theta_{1} \\
\phi_{2}=\left\{(a, b) \in R \times R:[a p]_{\theta_{1}}\right. & \left.=[b p]_{\theta_{1}}, \text { for every } p \in B\right\} .
\end{aligned}
$$

Then

1. $\phi$ is the greatest congruence on $\mathcal{D}$ having $\theta_{1}$ as Boolean part and $\mathcal{D} / \phi$ is separable;

2. $\left(\theta_{1}, \Delta_{\mathcal{R}}\right)$ is the smallest dynamic congruence on $\mathcal{D}$ having $\theta_{1}$ as Boolean part.

Proof. Let $\left(\theta_{1}, \theta_{2}\right) \in \operatorname{Cong} \mathcal{D}$. We show that $\phi$ defines a congruence on $\mathcal{D}$.

Trivially, $\phi_{2}$ is an equivalence relation. Let $(a, b),(c, d) \in \phi_{2}$. Then $[a p]_{\theta_{1}}=[b p]_{\theta_{1}}$ and $[c p]_{\theta_{1}}=[d p]_{\theta_{1}}$, for every $p \in B$. Immediately

$[(a \cup c) p]_{\theta_{1}}=[a p \vee c p]_{\theta_{1}}=[a p]_{\theta_{1}} \vee[c p]_{\theta_{1}}=[b p]_{\theta_{1}} \vee[d p]_{\theta_{1}}=[b p \vee d p]_{\theta_{1}}=[(b \cup d) p]_{\theta_{1}}$,

$[(a c) p]_{\theta_{1}}=[a(c p)]_{\theta_{1}}=[a]_{\theta_{2}}[c p]_{\theta_{1}}=[a]_{\theta_{2}}[d p]_{\theta_{1}}=[a(d p)]_{\theta_{1}}=[b(d p)]_{\theta_{1}}=[(b d) p]_{\theta_{1}}$,

$\left[a^{*} p\right]_{\theta_{1}}=\left[a^{*}\right]_{\theta_{2}}[p]_{\theta_{1}}=[a]_{\theta_{2}}^{*}[p]_{\theta_{1}}=\min \left\{[q]_{\theta_{1}}:[p]_{\theta_{1}} \vee[a]_{\theta_{2}}[q]_{\theta_{1}} \leq[q]_{\theta_{1}}\right\}$

$=\min \left\{[q]_{\theta_{1}}:[p]_{\theta_{1}} \vee[b]_{\theta_{2}}[q]_{\theta_{1}} \leq[q]_{\theta_{1}}\right\}=[b]_{\theta_{2}}^{*}[p]_{\theta_{1}}=\left[b^{*}\right]_{\theta_{2}}[p]_{\theta_{1}}=\left[b^{*} p\right]_{\theta_{1}}$, for every $p \in B$, and hence, $(a \cup c, b \cup d),(a c, b d)$ and $\left(a^{*}, b^{*}\right) \in \phi_{2}$.

Let $(a, b) \in \phi_{2}$ and $(p, q) \in \phi_{1}$. We have $[a]_{\theta_{2}}[p]_{\theta_{1}}=[b]_{\theta_{2}}[p]_{\theta_{1}}$. Since $[p]_{\theta_{1}}=[q]_{\theta_{1}}$ then $[a]_{\theta_{2}}[p]_{\theta_{1}}=[b]_{\theta_{2}}[q]_{\theta_{1}}$, and consequently, $[a p]_{\theta_{1}}=[b q]_{\theta_{1}}$. Therefore, $(a p, b q) \in \phi_{1}$.

It is clear that for any dynamic congruence $\alpha=\left(\theta_{1}, \alpha_{2}\right)$, we have, necessarily, $\alpha \subseteq \phi$. 


\section{Congruences on Dynamic Algebras}

Now suppose that $[a]_{\phi_{2}}[p]_{\phi_{1}}=[b]_{\phi_{2}}[p]_{\phi_{1}}$, for every $p \in B$. Then, for every $p \in B$,

$[a p]_{\phi_{1}}=[b p]_{\phi_{1}}$

$[a p]_{\theta_{1}}=[b p]_{\theta_{1}}$.

Consequently, $(a, b) \in \phi_{2}$, i.e., $[a]_{\phi_{2}}=[b]_{\phi_{2}}$, establishing the separability of $\mathcal{D} / \phi$.

DeFinition 4.3

Let $\theta_{1}$ and $\phi$ be as defined at Prop. 4.2. The relation $\phi$ is called the separating congruence of any $\theta \in \operatorname{Cong} \mathcal{D}$ having $\theta_{1}$ as Boolean part (or simply a separating congruence).

For any dynamic algebra $\mathcal{D}=(\mathcal{B}, \mathcal{R},\langle\rangle),\left(\Delta_{\mathcal{B}}, \cong_{\mathcal{R}}\right)(c f$. defined at $\S 1)$ is the separating congruence of $\left(\Delta_{\mathcal{B}}, \Delta_{\mathcal{R}}\right)$. Immediately, if $\mathcal{D}$ is separable, then $\left(\Delta_{\mathcal{B}}, \Delta_{\mathcal{R}}\right)$ is the unique dynamic congruence having $\Delta_{\mathcal{B}}$ as Boolean part.

Proposition 4.4

Let $\theta$ be a dynamic congruence on a dynamic algebra $\mathcal{D}$. Then $\mathcal{D} / \theta$ is separable if and only if $\theta$ is a separating congruence on $\mathcal{D}$.

\section{Dynamic Congruences and Dynamic Ideals}

From now on we are going to use only separable dynamic algebras $\mathcal{D}=(\mathcal{B}, \mathcal{R},\langle\rangle)$ where the regular part contains an element o satisfying o $p=0$, for every $p \in B$, and therefore, satisfying $\mathrm{o} a=\mathrm{o}, a \mathrm{o}=\mathrm{o}$ and $\mathrm{o} \cup a=a, a \cup \mathrm{o}=a$, for every $a \in R$. Such an algebra is called a separable dynamic algebra with zero.

We notice that every separable dynamic algebra $\mathcal{D}=(\mathcal{B}, \mathcal{R},\langle\rangle)$ can be enriched with a zero, by simply adding elements o, $i d$ and $a \cup i d$, for every $a \in R$, on the regular part of $\mathcal{D}$ (where $i d$ is defined by $\langle i d\rangle p=p$, for every $p \in B$ ).

Usually, the notion of $i d e a l$ in a given class of algebras is established so that the zero-classes of congruence relations are easily seen to be ideals. We will show, in this paragraph, that the class of separable dynamic algebras with zero is not a class with ideal determined congruences, [3], i.e., each ideal is not the zero-class of a unique congruence relation.

DeFinition 5.1

A (dynamic) ideal on a dynamic algebra with zero $\mathcal{D}=(\mathcal{B}, \mathcal{R},\langle\rangle)$ is a pair $I=\left(I_{1}, I_{2}\right)$ satisfying the following conditions

1. $I_{1}$ is a Boolean ideal on $\mathcal{B}$;

2. if $p \in I_{1}$ and $a \in R$ then $a p \in I_{1}$;

3. (a) $I_{2} \neq \emptyset$;

(b) if $a, b \in I_{2}, c, d \in R$ and $d \leq a$ then $a \cup b, a c, c a, d \in I_{2}$;

4. if $a \in I_{2}$ and $p \in B$ then $a p \in I_{1}$.

Such a subset $I_{2}$ of $R$ satisfying condition (3) is called an (action) ideal of $R$.

By definition of dynamic ideal, since o $\in R$ then $o \in I_{2}\left(o=\right.$ o $j \in I_{2}$, for some $\left.j \in I_{2} \neq \emptyset\right)$. We denote by $I d e \mathcal{D}$ the set of all ideals on a dynamic algebra with zero, $\mathcal{D}=(\mathcal{B}, \mathcal{R},\langle\rangle)$.

Similarly to the case of the congruences, in a separable dynamic algebra with zero $\mathcal{D}$, there are Boolean ideals that are not the Boolean part of any dynamic ideal on $\mathcal{D}$. A Boolean ideal $I_{1}$ is called dynamical on $\mathcal{D}=(\mathcal{B}, \mathcal{R},\langle\rangle)$ if there exists an action ideal $I_{2}$ on $\mathcal{R}$ such that $\left(I_{1}, I_{2}\right)$ is a dynamic ideal on $\mathcal{D}$. 
Proposition 5.2

Let $I_{1}$ be a Boolean ideal on a separable dynamic algebra with zero, $\mathcal{D}$. Then $I_{1}$ is a dynamical ideal on $\mathcal{D}$ if and only if $\left(I_{1},\{0\}\right)$ is a dynamic ideal.

We will present a separable dynamic algebra with zero where there exists a Boolean ideal $I_{1}$ which is not dynamical. Let $\mathcal{D}=(\mathcal{B}, \mathcal{R},\langle\rangle)$ be the dynamic algebra with $\mathcal{B}$ the Boolean algebra with four elements $\{0, p, q, 1\}$ and $R=\{a, o, i d\}$ where $a$, o and $i d$ are defined by $a 0=0, a p=a q=a 1=1,\langle 0\rangle s=0$ and $\langle i d\rangle s=s$, for every $s \in B$. The pair $\left(I_{1},\{0\}\right)$ with $I_{1}$ the Boolean ideal $\{0, p\}$, is not a dynamic ideal, since $a \in R, p \in I_{1}$ but $a p=1 \notin I_{1}$.

Proposition 5.3

Let $I_{1}$ be a dynamical ideal on a separable dynamic algebra with zero. Then

1. $\left(I_{1},\{\mathrm{o}\}\right)$ is the smallest dynamic ideal having $I_{1}$ as Boolean part;

2. ( $I_{1},\left\{a\right.$ : $a p \in I_{1}$, for every $\left.\left.p \in B\right\}\right)$ is the greatest dynamic ideal having $I_{1}$ as Boolean part.

DeFinITION 5.4

Let $I_{1}$ be a dynamical ideal on a separable dynamic algebra with zero, $\mathcal{D}=(\mathcal{B}, \mathcal{R},\langle\rangle)$, and let $F=\left(F_{1}, F_{2}\right)$ be defined by

$$
\begin{aligned}
& F_{1}:=I_{1} \\
& F_{2}:=\left\{a: a p \in I_{1}, \text { for every } p \in B\right\} .
\end{aligned}
$$

We say that $F$ is the determining ideal of any $I \in I d e \mathcal{D}$ having $I_{1}$ as Boolean part (or simply, a determining ideal).

\section{DeFinITION 5.5}

If $\theta=\left(\theta_{1}, \theta_{2}\right) \in \operatorname{Cong} \mathcal{D}$ where $\mathcal{D}=(\mathcal{B}, \mathcal{R},\langle\rangle)$ is a separable dynamic algebra with zero, we say that $\mathcal{I}(\theta):=\mathcal{I}^{\theta}=\left(\mathcal{I}_{1}^{\theta}, \mathcal{I}_{2}^{\theta}\right)$ defined by $\mathcal{I}_{1}^{\theta}:=\left\{p \in B: p \theta_{1} 0\right\}=[0]_{\theta_{1}}$

is the kernel of the congruence $\theta$.

$$
\mathcal{I}_{2}^{\theta}:=\left\{a \in R: a \theta_{2} \mathrm{O}\right\}=[\mathrm{o}]_{\theta_{2}}
$$

Proposition 5.6

The kernel of a congruence $\theta, \mathcal{I}(\theta)$, on a separable dynamic algebra with zero $\mathcal{D}=(\mathcal{B}, \mathcal{R},\langle\rangle)$, is an ideal on $\mathcal{D}$.

ProOF.

1. We know that $\mathcal{I}_{1}^{\theta}$ is a Boolean ideal .

2. If $a \in R$ and $p \in \mathcal{I}_{1}^{\theta}$, then $p \theta_{1} 0$ and $a \theta_{2} a$. Therefore, $(a p) \theta_{1}(a 0)$, i.e., $(a p) \theta_{1} 0$. So $a p \in \mathcal{I}_{1}^{\theta}$.

3. (a) $\mathcal{I}_{2}^{\theta} \neq \emptyset$, since o $\in \mathcal{I}_{2}^{\theta}$.

(b) If $a, b \in \mathcal{I}_{2}^{\theta}, c, d \in R$ and $d \leq a$ then $a \cup b, a c, c a, d \in \mathcal{I}_{2}^{\theta}$. In fact, if $a, b \in \mathcal{I}_{2}^{\theta}$ then $a \theta_{2} \mathrm{O}$ and $b \theta_{2} \mathrm{O}$, and therefore, $(a \cup b) \theta_{2}(\mathrm{o} \cup \mathrm{o})$, i.e., $(a \cup b) \theta_{2} \mathrm{O}$. Thus $a \cup b \in \mathcal{I}_{2}^{\theta}$. Since $c \theta_{2} c$ and $a \theta_{2} \mathrm{o}$ then $(a c) \theta_{2}(\mathrm{o} c)$ and $(c a) \theta_{2}(c \mathrm{o})$, i.e., $(a c) \theta_{2} \mathrm{o}$ and $(c a) \theta_{2} \mathrm{o}$. To this extend $a c$ and $c a$ are elements of $\mathcal{I}_{2}^{\theta}$. Since $d \theta_{2} d$ and $a \theta_{2}$ o then $(d \cup a) \theta_{2}(d \cup \mathrm{o})$, and therefore, $a \theta_{2} d$ (from $d \leq a$ we get $d \cup a=a$ ). But $a \theta_{2} \mathrm{O}$ and so $d \theta_{2} \mathrm{O}$. Therefore, $d \in \mathcal{I}_{2}^{\theta}$.

4. Let $a \in \mathcal{I}_{2}^{\theta}$ and $p \in B$. Then $a \theta_{2} \mathrm{O}$ and $p \theta_{1} p$, and therefore, $(a p) \theta_{1} \mathrm{o} p$, i.e., $(a p) \theta_{1} 0$. Immediately, $a p \in \mathcal{I}_{1}^{\theta}$.

Definition 5.7

The kernel of a dynamic homomorphism $h: \mathcal{D} \longrightarrow \mathcal{D}^{\prime}$, between dynamic algebras with zero, is the pair

$$
\left(\left\{p \in B: h_{1}(p)=0\right\},\left\{a \in R: h_{2}(a)=o\right\}\right) .
$$


Proposition 5.8

The kernel of a dynamic homomorphism $h: \mathcal{D} \longrightarrow \mathcal{D}^{\prime}$, between separable dynamic algebras with zero, is a dynamic ideal on $\mathcal{D}$.

Definition 5.9

If $I=\left(I_{1}, I_{2}\right)$ is a dynamic ideal on a separable dynamic algebra with zero $\mathcal{D}=(\mathcal{B}, \mathcal{R},\langle\rangle)$, we define $\mathcal{C}(I):=\mathcal{C}^{I}=\left(\mathcal{C}_{1}^{I}, \mathcal{C}_{2}^{I}\right)$ by

$p \mathcal{C}_{1}^{I} q$ if and only if $p \vee i=q \vee i$, for some $i \in I_{1}$,

$a \mathcal{C}_{2}^{I} b$ if and only if $a \cup j=b \cup j$, for some $j \in I_{2}$,

for $p, q \in B$ and $a, b \in R$.

Proposition 5.10

If $I=\left(I_{1}, I_{2}\right)$ is a dynamic ideal on a separable dynamic algebra with zero $\mathcal{D}=(\mathcal{B}, \mathcal{R},\langle\rangle)$, then $\mathcal{C}(\mathcal{I})$ is a congruence relation on $\mathcal{D}$.

Proof. (i) The relation $\mathcal{C}_{1}^{I}$ is a congruence relation on $\mathcal{B}$ (a known result in Boolean algebras).

(ii) Let us prove that $\mathcal{C}_{2}^{I}$ is an equivalence relation on $\mathcal{R}$.

(a) For every $a \in R, a \mathcal{C}_{2}^{I} a$ since $a \cup \mathrm{o}=a \cup \mathrm{o}$, with $\mathrm{o} \in I_{2}$.

(b) Obviously, for $a, b \in R$, if $a \mathcal{C}_{2}^{I} b$ then $b \mathcal{C}_{2}^{I} a$.

(c) For $a, b \in R$, if $a \mathcal{C}_{2}^{I} b$ and $b \mathcal{C}_{2}^{I} c$ then $a \mathcal{C}_{2}^{I} c$. In fact, if $a \mathcal{C}_{2}^{I} b$ and $b \mathcal{C}_{2}^{I} c$ then $a \cup j=b \cup j$ and $b \cup k=c \cup k$ for some $j$ and $k$, both in $I_{2}$. Therefore,

$$
\begin{aligned}
a \cup(j \cup k) & =b \cup j \cup k \\
& =c \cup k \cup j \\
& =c \cup(j \cup k) .
\end{aligned}
$$

Since $j \cup k \in I_{2}$, then $a \mathcal{C}_{2}^{I} c$.

(iii) Now we prove that, for $a, b \in R$ and $p, q \in B$, if $a \mathcal{C}_{2}^{I} b$ and $p \mathcal{C}_{1}^{I} q$ then $(a p) \mathcal{C}_{1}^{I}(b q)$. Let us assume that $p \mathcal{C}_{1}^{I} q$ and $a \mathcal{C}_{2}^{I} b$. Then $p \vee i=q \vee i$ for some $i \in I_{1}$ and $a \cup j=b \cup j$ for some $j \in I_{2}$ (and therefore $a q \vee j q=b q \vee j q$ ). Since $p \vee i=q \vee i$ then $a p \vee a i=a q \vee a i$ and moreover $a p \vee$ $a i \vee j q=a q \vee a i \vee j q$. So

$$
\begin{aligned}
a p \vee(a i \vee j q) & =a q \vee j q \vee a i \\
& =b q \vee j q \vee a i \\
& =b q \vee(a i \vee j q) .
\end{aligned}
$$

Since $a i \vee j q \in I_{1}$ then $(a p) \mathcal{C}_{1}^{I}(b q)$.

(iv) It remains to be proved that, for $a, b \in R$, if $a \mathcal{C}_{2}^{I} b$ and $c \mathcal{C}_{2}^{I} d$ then $(a \cup c) \mathcal{C}_{2}^{I}(b \cup d)$, $(a c) \mathcal{C}_{2}^{I}(b d)$ and $\left(a^{*}\right) \mathcal{C}_{2}^{I}\left(b^{*}\right)$. Let us admit that $a \mathcal{C}_{2}^{I} b$ and $c \mathcal{C}_{2}^{I} d$. Then there exist $j, k$ in $I_{2}$ such that $a \cup j=b \cup j$ and $c \cup k=d \cup k$.

(a) So, $a \cup j \cup c \cup k=b \cup j \cup d \cup k$, and therefore, $(a \cup c) \cup(j \cup k)=(b \cup d) \cup(j \cup k)$. Since $j \cup k \in I_{2}$ then $(a \cup c) \mathcal{C}_{2}^{I}(b \cup d)$.

(b) From $c \cup k=d \cup k$ we get $a(c \cup k)=a(d \cup k)$, i.e., $a c \cup a k=a d \cup a k$. (We note that we are working with a separable dynamic algebra, and, therefore, we are allowed to say that $a(b \cup c)=a b \cup a c$, for every $a, b, c \in R$.)

Hence,

$$
\begin{aligned}
a c \cup(a k \cup j d) & =a d \cup a k \cup j d \\
& =(a \cup j) d \cup a k \\
& =(b \cup j) d \cup a k \\
& =b d \cup(j d \cup a k) .
\end{aligned}
$$

From $j d \cup a k \in I_{2}$ we get $(a c) \mathcal{C}_{2}^{I}(b d)$. 
(c) Since $a \cup j=b \cup j$ then $a p \vee j p=b p \vee j p$, for every $p \in B$. In particular, for $b^{*} p$ we have $a\left(b^{*} p\right) \vee j\left(b^{*} p\right)=b\left(b^{*} p\right) \vee j\left(b^{*} p\right)$. Then $a b^{*} p \vee j b^{*} p \vee a^{*} j b^{*} p=b b^{*} p \vee j b^{*} p \vee a^{*} j b^{*} p$.

Condition (5a) of dynamic algebras yields $q \leq q \vee a a^{*} q \leq a^{*} q$ and consequently $q \vee a^{*} q=$ $a^{*} q$, for every $q \in B$. For $q=j b^{*} p$, we get $j b^{*} p \vee a^{*} j b^{*} p=a^{*} j b^{*} p$.

Therefore

$$
\begin{array}{rlrl}
a b^{*} p \vee a^{*} j b^{*} p & =b b^{*} p \vee a^{*} j b^{*} p . & \\
a\left(b^{*} p \vee a^{*} j b^{*} p\right) & =a b^{*} p \vee a a^{*} j b^{*} p & \\
& \leq a b^{*} p \vee a^{*} j b^{*} p & & (b y(5 a)) \\
& =b b^{*} p \vee a^{*} j b^{*} p & & (b y(5.1)) \\
& \leq b^{*} p \vee a^{*} j b^{*} p . & & (b y(5 a))
\end{array}
$$

If we take $r:=b^{*} p \vee a^{*} j b^{*} p$, we have $a r \leq r$. Since $p \leq b^{*} p$ (for every $p \in B$ ) and $b^{*} p \leq r$ then $p \leq r$. Therefore $p \vee a r \leq r$, and so $r \in a ! p$, and consequently, $a^{*} p \leq r$, i.e., $a^{*} p \leq b^{*} p \vee a^{*} j b^{*} p$. Interchanging $a$ and $b$, we get $b^{*} p \leq a^{*} p \vee b^{*} j a^{*} p$. Consequently, we have $a^{*} p \leq b^{*} p \vee a^{*} j b^{*} p \vee b^{*} j a^{*} p$ and $b^{*} p \leq a^{*} p \vee b^{*} j a^{*} p \vee a^{*} j b^{*} p$. Letting $l:=a^{*} j b^{*} \cup b^{*} j a^{*}$ we get $a^{*} p \leq b^{*} p \vee l p$ and $b^{*} p \leq a^{*} p \vee l p$, and hence $a^{*} p \vee l p \leq b^{*} p \vee l p$ and $b^{*} p \vee l p \leq a^{*} p \vee l p$. Therefore, $a^{*} p \vee l p=b^{*} p \vee l p$, for every $p \in B$. So, there exists $l \in I_{2}$ such that $a^{*} \cup l=b^{*} \cup l$ which means that $\left(a^{*}\right) \mathcal{C}_{2}^{I}\left(b^{*}\right)$.

Proposition 5.11

If $I=\left(I_{1}, I_{2}\right)$ is an ideal on a separable dynamic algebra with zero, then $\mathcal{I}(\mathcal{C}(I))=I$.

Proof. We pretend to show that $[0]_{\mathcal{C}_{1}^{I}}=I_{1}$ and $[o]_{\mathcal{C}_{2}^{I}}=I_{2}$. The first assertion is a well known Boolean fact.

(i) We begin by proving that $[\mathrm{o}]_{\mathcal{C}_{2}^{I}} \subseteq I_{2}$. For $a \in[\mathrm{o}]_{\mathcal{C}_{2}^{I}}$ we have $a \cup j=\mathrm{o} \cup j$, for some $j \in I_{2}$. Then, $a \cup j=j$, and therefore, $a \leq j$. Since $j \in I_{2}$ then $a \in I_{2}$.

(ii) Now, we prove that $I_{2} \subseteq[\mathrm{o}]_{\mathcal{C}_{2}^{I}}$. Let $a \in I_{2}$. From $a \cup a=\mathrm{o} \cup a$ we get $a \in[\mathrm{o}]_{\mathcal{C}_{2}^{I}}$.

Proposition 5.12

On a separable dynamic algebra with zero, a dynamic ideal is a determining ideal if and only if it is the kernel of a separating congruence.

Proof. Let $I=\left(I_{1}, I_{2}\right)$ be a dynamic ideal on a separable dynamic algebra with zero $\mathcal{D}=(\mathcal{B}, \mathcal{R},\langle\rangle)$. By Prop.5.11 there exists a dynamic congruence $\theta$ such that $I=\mathcal{I}(\theta)$. Let $\phi$ be the separating congruence of $\theta$. We have $\phi_{1}=\theta_{1}$ and $\phi_{2}=\left\{(a, b): a p \theta_{1} b p\right.$ for every $p \in$ $B\}$. So, $\mathcal{I}_{1}^{\phi}=\mathcal{I}_{1}^{\theta}=I_{1}$ and $\mathcal{I}_{2}^{\phi}=\left\{a: a \phi_{2} \mathrm{O}\right\}$. Then, we have to prove that $\left\{a: a \phi_{2} \mathrm{O}\right\}=\{a: a p \in$ $I_{1}$, for every $\left.p \in B\right\}$. But, for every $a \in R$, we have $a \phi_{2} \mathrm{O}$ if and only if $a p \theta_{1} 0$, for every $p \in B$, i.e., $a p \in[0]_{\theta 1}=I_{1}$.

As the following example will show, there exist separable dynamic algebras with zero where dynamic congruences may or may not have the same kernel of its separating congruences. So, we can have different congruences with the same kernel. It is a well known fact that every Boolean congruence is determined by its kernel. That is not the case for dynamic congruences.

EXAMPLE 5.13

Let $\quad \mathcal{D}_{1}=(\mathcal{P}(S),\{a, b, c, d$, o $\},\langle\rangle), \quad$ for $\quad S=\{1,2,3\}, \quad a=\{(1,1)\}, \quad b=\{(2,2)\}, \quad c=\{(1,1)$, $(2,2)\}, d=\{(1,1),(2,2),(3,3)\}$ and $o=\emptyset$. We begin with a short study on the 
structure $\mathcal{D}_{1}$, that will be useful later on. We note that $\mathcal{D}_{1}$ is a well defined Kripke structure. In fact,

$$
\begin{array}{lllll}
a\{1\}=\{1\} & b\{1\}=\varnothing & c\{1\}=\{1\} & d\{1\}=\{1\} & o\{1\}=\emptyset \\
a\{2\}=\emptyset & b\{2\}=\{2\} & c\{2\}=\{2\} & d\{2\}=\{2\} & o\{2\}=\emptyset \\
a\{3\}=\emptyset & b\{3\}=\varnothing & c\{3\}=\varnothing & d\{3\}=\{3\} & o\{3\}=\emptyset \\
& & & \\
a \cup a=a & b \cup b=b & c \cup c=c & d \cup d=d & \mathrm{o} \cup \mathrm{o}=\mathrm{o} \\
a \cup b=c & b \cup c=c & c \cup d=d & d \cup \mathrm{o}=d & \\
a \cup c=c & b \cup d=d & c \cup \mathrm{o}=c & \\
a \cup d=d & b \cup \mathrm{o}=b & & & \\
a \cup \mathrm{o}=a & & & \\
a a=a & b a=\mathrm{o} & c a=a & d a=a & \mathrm{o} a=\mathrm{o} \\
a b=\mathrm{o} & b b=b & c b=b & d b=b & \mathrm{o} b=\mathrm{o} \\
a c=a & b c=b & c c=c & d c=c & \mathrm{o} c=\mathrm{o} \\
a d=a & b d=b & c d=c & d d=d & \mathrm{o} d=\mathrm{o} \\
a \mathrm{o}=\mathrm{o} & b \mathrm{o}=\mathrm{o} & c \mathrm{o}=\mathrm{o} & d \mathrm{o}=\mathrm{o} & \mathrm{oo}=\mathrm{o} \\
& & & & \\
a^{*}=d & b^{*}=d & c^{*}=d & d^{*}=d & 0^{*}=d
\end{array}
$$

Let $\theta=\mathcal{C}(I)$ and $\beta=\mathcal{C}(J)$ with $I=(\{\varnothing,\{1\},\{3\},\{1,3\}\},\{0, a\})$ and $J=(\{\varnothing,\{1\}\},\{0\})$. Since, $I$ and $J$ are dynamic ideals on $\mathcal{D}_{1}$ then $\theta$ and $\beta$ are congruences with classes

$$
\begin{array}{ll}
{[\emptyset]_{\theta_{1}}=\{\emptyset,\{1\},\{3\},\{1,3\}\}} & {[\emptyset]_{\beta_{1}}=\{\emptyset,\{1\}\}} \\
{[\{2\}]_{\theta_{1}}=\{\{2\},\{1,2\},\{2,3\},\{1,2,3\}\}} & {[\{2\}]_{\beta_{1}}=\{\{2\},\{1,2\}\}} \\
{[0]_{\theta_{2}}=\{0, a\}} & {[\{3\}]_{\beta_{1}}=\{\{3\},\{1,3\}\}} \\
{[b]_{\theta_{2}}=\{b, c\}} & {[\{2,3\}]_{\beta_{1}}=\{\{2,3\},\{1,2,3\}\}} \\
{[d]_{\theta_{2}}=\{d\} .} & {[m]_{\beta_{2}}=\{m\}, \text { for every } m \in\{a, b, c, d, o\} .}
\end{array}
$$

The separating congruences of $\theta$ and $\beta$ are, respectively, $\delta$ and $\phi$ with classes

$$
\begin{array}{ll}
{[t]_{\delta_{1}}=[t]_{\theta_{1}}, \text { for every } t \in \mathcal{P}(S)} & {[t]_{\phi_{1}}=[t]_{\beta_{1}} \text {, for every } t \in \mathcal{P}(S)} \\
{[0]_{\delta_{2}}=\{0, a\}} & {[0]_{\phi_{2}}=\{0, a\}} \\
{[b]_{\delta_{2}}=\{b, c, d\}} & {[b]_{\phi_{2}}=\{b, c\}} \\
& {[d]_{\phi_{2}}=\{d\}}
\end{array}
$$

So, while the kernel of $\beta$ is $(\{\varnothing,\{1\}\},\{0\})$ and the kernel of $\phi$ is $(\{\varnothing,\{1\}\},\{0, a\})$, the congruences $\theta$ and $\delta$ have the same kernel $(\{\varnothing,\{1\},\{3\},\{1,3\}\},\{0, a\})$. 
To any dynamic ideal $I$, since $\mathcal{I}(\mathcal{C}(I))=I$, the class of all dynamic congruences having $I$ as its kernel is not empty. On the other hand, the intersection of all these dynamic congruences is always a congruence with kernel equal to $I$. Therefore, we can establish the following definition.

DeFINITION 5.14

The congruence generated by a dynamic ideal $I$, is the smallest congruence with kernel equal to $I$.

Proposition 5.15

Let $I=\left(I_{1}, I_{2}\right)$ be an ideal on a separable dynamic algebra with zero. Then $\mathcal{C}(I)$ is the congruence generated by $I$. In particular, $\mathcal{C}(\mathcal{I}(\theta)) \subseteq \theta$, for every dynamic congruence $\theta$.

Proof. Let $I=\left(I_{1}, I_{2}\right)$ be an ideal on a separable dynamic algebra with zero $\mathcal{D}=(\mathcal{B}, \mathcal{R},\langle\rangle)$. Let $\delta \in \operatorname{Cong} \mathcal{D}$ such that $\mathcal{I}(\delta)=I$. If $(a, b) \in \mathcal{C}_{2}^{I}$, then $a \cup j=b \cup j$, for some $j \in I_{2}$. By assumption, $I_{2}=\mathcal{I}_{2}^{\delta}$, and so, $a \cup j=b \cup j$, for some $j \in \mathcal{I}_{2}^{\delta}$, i.e., $a \cup j=b \cup j$, for some $j$ such that $j \delta_{2} \mathrm{O}$. Since $j \delta_{2} \mathrm{O}$ we have $(a \cup j) \delta_{2}(a \cup \mathrm{o})$ and $(b \cup j) \delta_{2}(b \cup \mathrm{o})$. Then $(a \cup j) \delta_{2} a$ and $(b \cup j) \delta_{2} b$, and from $a \cup j=b \cup j$, we get $a \delta_{2} b$, i.e., $(a, b) \in \delta_{2}$. Similarly, we prove that $\mathcal{C}_{1}^{I} \subseteq \delta_{1}$. (Actually, $\mathcal{C}_{1}^{I}=\delta_{1}$, a known result in Boolean algebras.)

We could be led to ask if, for every congruence $\theta=\left(\theta_{1}, \theta_{2}\right)$ on a separable dynamic algebra with zero, we have $\mathcal{C}(\mathcal{I}(\theta))=\theta$. In general, that is not the fact.

EXAMPLE 5.16

Recovering Ex.5.13, we have $\mathcal{C}(\mathcal{I}(\delta)) \neq \delta$, since $\mathcal{I}_{2}^{\delta}=\{\mathrm{o}, a\}=[\mathrm{o}]_{\theta_{2}}$, and therefore, $\mathcal{C}_{2}^{\mathcal{I}(\delta)}=\theta_{2} \neq \delta_{2}$.

It is immediate to establish, as a consequence of Prop.5.11, a necessary and sufficient condition on $\theta$ to have the equality $\mathcal{C}(\mathcal{I}(\theta))=\theta$.

Proposition 5.17

Let $\theta=\left(\theta_{1}, \theta_{2}\right) \in \operatorname{Cong} \mathcal{D}$ where $\mathcal{D}$ is a separable dynamic algebra with zero. Then $\mathcal{C}(\mathcal{I}(\theta))=\theta$ if and only if $\theta=\mathcal{C}(I)$ for some ideal $I$ on $\mathcal{D}$, i.e., $\mathcal{C}(\mathcal{I}(\theta))=\theta$ if and only if $\theta \in \mathcal{C}(\mathcal{I}$ de $\mathcal{D})$.

\section{The lattice IdeD and a Galois Connection between $\operatorname{Cong} \mathcal{D}$ and Ide $\mathcal{D}$}

We intend to insert a lattice structure into $I d e \mathcal{D}$, the set of all dynamic ideals on a separable dynamic algebra with zero, $\mathcal{D}$. To do so we need to define, for arbitrary dynamic ideals $I$ and $J, I \wedge_{\mathcal{D}} J$ and $I \vee_{\mathcal{D}} J$. It is immediate to put $I \wedge_{\mathcal{D}} J=\left(I_{1} \cap J_{1}, I_{2} \cap J_{2}\right)$. Once again the disjunction requires some attention. Surprisingly, we are able to prove the following proposition. We notice that we use the fact that the subset of actions is a union of action ideals and not an arbitrary subset of actions.

We denote by $\langle X\rangle_{\mathcal{A}}$ the ideal generated by a subset $X$ of any (homogeneous or heterogeneous) algebra $\mathcal{A}$, i.e., the intersection of all ideals $I$ on $\mathcal{A}$ containing $X$,

$$
\langle X\rangle_{\mathcal{A}}:=\cap\{I: I \text { ideal on } A \text { and } X \subseteq I\} .
$$

\section{Proposition 6.1}

Let $I=\left(I_{1}, I_{2}\right)$ and $J=\left(J_{1}, J_{2}\right)$ be elements of $I d e \mathcal{D}$, with $\mathcal{D}=(\mathcal{B}, \mathcal{R},\langle\rangle)$ a separable dynamic algebra with zero. We have:

$$
\begin{array}{r}
\left\langle I_{1} \cup J_{1}\right\rangle_{\mathcal{B}}=\left\{p \in B: p \leq p_{1} \vee \cdots \vee p_{n}, \text { for some integer } n \geq 1\right. \\
\left.\quad \text { and some } p_{i} \in I_{1} \cup J_{1}, i=1, \cdots, n\right\} \\
\left\langle I_{2} \cup J_{2}\right\rangle_{\mathcal{R}}=\left\{a \in R: a \leq a_{1} \cup \cdots \cup a_{n}, \text { for some integer } n \geq 1\right. \\
\text { and some } \left.a_{i} \in I_{2} \cup J_{2}, i=1, \cdots, n\right\}
\end{array}
$$




\section{Congruences on Dynamic Algebras}

Proof. We only have to prove the second identity since the first is a well known Boolean result [2]. We want to show that, for $X=\left\{a \in R\right.$ : $a \leq a_{1} \cup \ldots \cup a_{n}$ for some integer $n \geq 1$ and some $\left.a_{i} \in I_{2} \cup J_{2}, i=1, \ldots, n\right\}$, we have:

(i) $X$ is an (action) ideal of $\mathcal{R}$;

(ii) $I_{2} \cup J_{2} \subseteq X$;

(iii) if $Y$ is an (action) ideal of $\mathcal{R}$ and $I_{2} \cup J_{2} \subseteq Y$, then $X \subseteq Y$.

Notice that $X$ is nonempty, since o $\leq a_{i}$ for any $a_{i} \in I_{2} \cup J_{2}$. Now, let $a, b \in X, c, d \in R$ and $d \leq a$. Then $a \leq a_{1} \cup \ldots \cup a_{n}$, for some integer $n \geq 1$ and some $a_{i} \in I_{2} \cup J_{2}, i=1, \ldots, n$, and $b \leq b_{1} \cup \ldots \cup b_{m}$ for some integer $m \geq 1$ and some $b_{j} \in I_{2} \cup J_{2}, j=1, \ldots, m$.

So, $\quad a \cup b \leq a_{1} \cup \ldots \cup a_{n} \cup b_{1} \cup \ldots \cup b_{m} \quad d \leq a \leq a_{1} \cup \ldots \cup a_{n}, \quad c a \leq c a_{1} \cup \ldots \cup c a_{n} \quad$ and $a c \leq a_{1} c \cup \ldots \cup a_{n} c$. Since for $i=1, \ldots, n$ and $j=1, \ldots, m, a_{i}, b_{j}, c a_{i}$ and $a_{i} c$ are elements of $I_{2} \cup J_{2}$, we get $a \cup b, d, c a$ and $a c$ in $\left\langle I_{2} \cup J_{2}\right\rangle_{\mathcal{R}}$. Therefore, $X$ is an (action) ideal of $\mathcal{R}$. It is straightforward that $I_{2} \cup J_{2} \subseteq X$. Let $a \in X$ and $Y$ be an (action) ideal of $\mathcal{R}$ such that $I_{2} \cup J_{2}$ $\subseteq Y$. Then $a \leq a_{1} \cup \ldots \cup a_{n}$, for some integer $n \geq 1$ and some $a_{i} \in I_{2} \cup J_{2} \subseteq Y, i=1, \ldots, n$. But $Y$ is an (action) ideal of $\mathcal{R}$ and $a_{i} \in Y$ for $i=1, \ldots, n$ so $a_{1} \cup \ldots \cup a_{n} \in Y$. Therefore $a \leq s$ with $s$ $=a_{1} \cup \ldots \cup a_{n} \in Y$. Since $Y$ is an (action) ideal of $\mathcal{R}$ we get $a \in Y$.

For $\mathcal{D}=(\mathcal{B}, \mathcal{R},\langle\rangle)$ dynamic algebra, $I \subseteq B$ and $J \subseteq R$ we write $J I$ to represent the set $J I:=\{a p: a \in J$ and $p \in I\}$.

Proposition 6.2

For $I=\left(I_{1}, I_{2}\right)$ and $J=\left(J_{1}, J_{2}\right)$ elements of $I d e \mathcal{D}$, with $\mathcal{D}=(\mathcal{B}, \mathcal{R},\langle\rangle)$ a separable dynamic algebra with zero, we have

$$
\begin{aligned}
& R\left(\left\langle I_{1} \cup J_{1}\right\rangle_{\mathcal{B}}\right) \subseteq\left\langle I_{1} \cup J_{1}\right\rangle_{\mathcal{B}}, \\
& \left(\left\langle I_{2} \cup J_{2}\right\rangle_{\mathcal{R}}\right) B \subseteq\left\langle I_{1} \cup J_{1}\right\rangle_{\mathcal{B}} .
\end{aligned}
$$

Proof. Let $a \in R$ and $p \in\left\langle I_{1} \cup J_{1}\right\rangle_{\mathcal{B}}$. By Prop.6.1, there exist an integer $n \geq 1$ and $p_{i} \in I_{1} \cup J_{1}$, $i=1, \ldots, n$ such that $p \leq p_{1} \vee \ldots \vee p_{n}$. Therefore $a p \leq a p_{1} \vee \ldots \vee a p_{n}$ and, since either $p_{i} \in I_{1}$ or $p_{i} \in J_{1}$ for $i=1, \ldots, n$, we have either $a p_{i} \in I_{1}$ or $a p_{i} \in J_{1}$ and then $a p \in\left\langle I_{1} \cup J_{1}\right\rangle_{\mathcal{B}}$.

Now, let $d \in\left\langle I_{2} \cup J_{2}\right\rangle_{\mathcal{R}}$ and $q \in B$. By Prop.6.1, there exist an integer $m \geq 1$ and $d_{i} \in I_{2} \cup$ $J_{2}, i=1, \ldots, m$ such that $d \leq d_{1} \cup \ldots \cup d_{m}$. Therefore $d q \leq d_{1} q \vee \ldots \vee d_{m} q$ and, since either $d_{i} \in I_{2}$ or $d_{i} \in J_{2}$ for $i=1, \ldots, m$, we have either $d_{i} q \in I_{1}$ or $d_{i} q \in J_{1}$ and then $d q \in\left\langle I_{1} \cup J_{1}\right\rangle_{\mathcal{B}}$.

Therefore, we are able to establish that the structure $\mathcal{I} d e \mathcal{D}=\left(\operatorname{Ide} \mathcal{D}, \wedge_{\mathcal{D}}, \vee_{\mathcal{D}}\right)$ where, for every $I=\left(I_{1}, I_{2}\right), J=\left(J_{1}, J_{2}\right) \in I d e \mathcal{D}$, the operations are defined by

$$
\begin{aligned}
& I \wedge_{\mathcal{D}} J:=I \cap J=\left(I_{1} \cap J_{1}, I_{2} \cap J_{2}\right) \\
& I \vee_{\mathcal{D}} J:=\langle I \cup J\rangle_{\mathcal{D}}=\left(\left\langle I_{1} \cup J_{1}\right\rangle_{B},\left\langle I_{2} \cup J_{2}\right\rangle_{R}\right)
\end{aligned}
$$

is a lattice, called the lattice of ideals of $\mathcal{D}$.

DeFinition 6.3

[1] A Galois-connection between the partially-ordered sets $\left(A, \leq_{A}\right)$ and $\left(B, \leq_{B}\right)$ is a pair of functions $(\mathcal{C}, \mathcal{I}), \mathcal{C}: A \longrightarrow B, \mathcal{I}: B \longrightarrow A$ satisfying, for all $x \in A$ and $y \in B$, the following restriction

$$
\mathcal{C}(x) \leq_{B} y \text { iff } x \leq_{A} \mathcal{I}(y) .
$$


THEOREM 6.4

The pair of maps $\mathcal{C}: I d e \mathcal{D} \longrightarrow \operatorname{Cong} \mathcal{D}$ (that for each $I \in I d e \mathcal{D}$ assigns the congruence $\mathcal{C}(I)$ ) and $\mathcal{I}: \operatorname{Cong} \mathcal{D} \longrightarrow I d e \mathcal{D}$ (that for each $\theta \in C o n g \mathcal{D}$ assigns the ideal $\mathcal{I}(\theta)$ ) defines a Galoisconnection $(\mathcal{C}, \mathcal{I})$, between the lattices $\operatorname{Ide} \mathcal{D}$ and $\operatorname{Cong} \mathcal{D}$.

Proof. Let $I$ be in $I d e \mathcal{D}$ and $\theta$ be in Cong $\mathcal{D}$. If $\mathcal{C}(I) \subseteq \theta$ then $\mathcal{I}(\mathcal{C}(I)) \subseteq \mathcal{I}(\theta)$, and by Prop.5.11 we have $I \subseteq \mathcal{I}(\theta)$. Now, if $I \subseteq \mathcal{I}(\theta)$ then $\mathcal{C}(I) \subseteq \mathcal{C}(\mathcal{I}(\theta))$. By Prop.5.15 we conclude $\mathcal{C}(I) \subseteq \theta$.

As an immediate consequence of 7.33 of [1] we have the following results.

Corollary 6.5

Let $\mathcal{D}$ be a separable dynamic algebra with zero. Then

1. for each $I \in I d e \mathcal{D}, \mathcal{C}(I)$ is the least congruence $\theta$ such that $I \subseteq \mathcal{I}(\theta)$;

2. for each $\theta \in \operatorname{Cong} \mathcal{D}, \mathcal{I}(\theta)$ is the greatest ideal $I$ such that $\mathcal{C}(I) \subseteq \theta$.

\section{Additive Ideals}

We already know that, in general, there are several dynamic congruences with the same kernel. The aim of this section is to study conditions under which a dynamic congruence is determined by its kernel. For that purpose we introduce the concept of additive ideal.

\section{DeFinition 7.1}

An ideal $I=\left(I_{1}, I_{2}\right)$ on a separable dynamic algebra with zero $\mathcal{D}=(\mathcal{B}, \mathcal{R},\langle\rangle)$ is said to be an additive ideal if, for every $a, b \in R$ such that $a p+b p \in I_{1}$, for every $p \in B$, there exists a $c \in I_{2}$ such that $a p+b p=c p$, for every $p \in B$.

On a separable dynamic algebra with zero, the pair $(\{0\},\{0\})$ is an additive ideal. Actually, it is the unique ideal with $\{0\}$ as Boolean part.

The following table distinguishes the ideals on the dynamic algebra $\mathcal{D}_{1}$, given at Ex.5.13, into additive and non-additive ideals.

$$
\text { Additive Ideals } I=\left(I_{1}, I_{2}\right)
$$

$$
\begin{aligned}
& I_{1}=\{\emptyset\} ; I_{2}=\{\mathrm{o}\} \\
& I_{1}=\{\emptyset,\{1\}\} ; I_{2}=\{\mathrm{o}, a\} \\
& I_{1}=\{\emptyset\{2\}\} ; I_{2}=\{\mathrm{o}, b\} \\
& I_{1}=\{\emptyset,\{1\},\{2\},\{1,2\}\} ; I_{2}=\{\mathrm{o}, a, b, c\}
\end{aligned}
$$

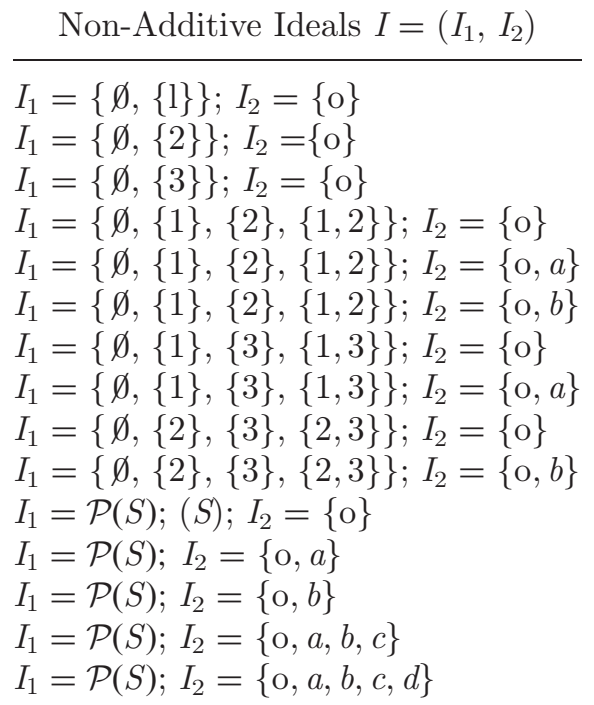


Theorem 7.2

Let $\theta=\left(\theta_{1}, \theta_{2}\right) \in \operatorname{Cong} \mathcal{D}$ where $\mathcal{D}=(\mathcal{B}, \mathcal{R},\langle\rangle)$ is a separable dynamic algebra with zero. If $\mathcal{I}(\theta)$ is an additive ideal, then

1. $\mathcal{C}(\mathcal{I}(\theta))=\theta$;

2. $\theta$ is determined by its kernel.

Proof. (1) We intend to show that $\mathcal{C}(\mathcal{I}(\theta))=\theta$, i.e., that $\mathcal{C}_{1}^{\mathcal{I}(\theta)}=\theta_{1}$ and $\mathcal{C}_{2}^{\mathcal{I}(\theta)}=\theta_{2}$. We are going to prove only that $\mathcal{C}_{2}^{\mathcal{I}(\theta)}=\theta_{2}$ (since $\mathcal{C}_{1}^{\mathcal{I}(\theta)}=\theta_{1}$ is a known result in Boolean algebras).

(i) We begin by showing that $\mathcal{C}_{2}^{\mathcal{I}(\theta)} \subseteq \theta_{2}$. Let $(a, b) \in \mathcal{C}_{2}^{\mathcal{I}(\theta)}$. Then $a \cup j=b \cup j$, for some $j \in \mathcal{I}_{2}^{\theta}$. Since $j \theta_{2} \mathrm{O}$ then $(a \cup j) \theta_{2}(a \cup o)$ and $(b \cup j) \theta_{2}(b \cup o)$, and therefore, $(a \cup j) \theta_{2} a$ and $(b \cup j) \theta_{2} b$. Since $a \cup j=b \cup j$ then $a \theta_{2} b$, i.e., $(a, b) \in \theta_{2}$.

(ii) Now we prove that $\theta_{2} \subseteq \mathcal{C}_{2}^{\mathcal{I}(\theta)}$. For $(a, b) \in \theta_{2}$ we have $a p \theta_{1} b p$, for every $p \in B$. And therefore, $(a p+b p) \theta_{1} 0$, i.e., $a p+b p \in[0]_{\theta_{1}}=\mathcal{I}_{1}^{\theta}$. Since $\mathcal{I}(\theta)$ is an additive ideal, there exists a $c \in \mathcal{I}_{2}^{\theta}$ such that $a p+b p=c p$, for every $p \in B$. Therefore $a p \vee c p=b p \vee c p$, and so, $(a \cup c) p=(b \cup c) p$, for every $p \in B$. The separability of $\mathcal{D}$ brings $a \cup c=b \cup c$, with $c \in \mathcal{I}_{2}^{\theta}$, i.e., $(a, b) \in \mathcal{C}_{2}^{\mathcal{I}(\theta)}$.

(2) We have to prove that $\theta$ is the unique congruence having $\mathcal{I}(\theta)$ as kernel. Let $\delta$ be a congruence on $\mathcal{D}$ such that $\mathcal{I}(\delta)=\mathcal{I}(\theta)$. Then $\mathcal{I}(\delta)$ is an additive ideal and, by (1), we have $\delta=\mathcal{C}(\mathcal{I}(\delta))=\mathcal{C}(\mathcal{I}(\theta))=\theta$.

A counterexample to the reciprocal result of Th.7.2(1) is given by a congruence generated by a non-additive ideal.

Proposition 7.3

Let $I=\left(I_{1}, I_{2}\right)$ and $I^{\prime}=\left(I_{1}, I_{2}^{\prime}\right)$ be ideals on a separable dynamic algebra with zero $\mathcal{D}=(\mathcal{B}, \mathcal{R},\langle\rangle)$. If $I$ is an additive ideal on $\mathcal{D}$ then

1. $I \subseteq I$;

2. $I$ is a determining ideal on $\mathcal{D}$.

Proof. 1. For $a \in I_{2}^{\prime}$, we have $a p \in I_{1}$, for every $p \in B$. Since $I$ is additive and $a p+o p \in I_{1}$, for every $p \in B$ (we recall that $a p+\mathrm{o} p=a p$ ), there exists a $c \in I_{2}$ such that $a p+\mathrm{o} p=c p$, for every $p \in B$. Therefore $a p=c p$, for every $p \in B$ and, since $\mathcal{D}$ is separable, we have $a=c$, showing that $a \in I_{2}$.

2. By 1., if there exists an additive ideal with Boolean part $I_{1}$, then it is the greatest (and the unique) additive ideal with Boolean part $I_{1}$. By Prop.5.3 the determining ideal of any ideal having $I_{1}$ as Boolean part is the greatest ideal with this property.

By recalling Ex.5.13, $(\{\emptyset,\{1\},\{3\},\{1,3\}\},\{0, a\})$ is an example of a non-additive determining ideal. Therefore, a determining ideal is not necessarily an additive ideal.

Corollary 7.4

If $I=\left(I_{1}, I_{2}\right)$ is an additive ideal on a separable dynamic algebra with zero $\mathcal{D}=(\mathcal{B}, \mathcal{R},\langle\rangle)$, then $\mathcal{C}(I)$ is a separating congruence.

\section{References}

[1] B.A. Davey and H.A. Priestley. Introduction to Lattices and Order, Cambridge University Press, Second Edition, 2002.

[2] G. Gratzer. General Lattice Theory, Birkhauser Verlag, 1998. 
[3] H. Peter Gumm and Aldo Ursini. Ideals in universal algebras, Algebra Universalis, 19, $45-54,1984$.

[4] D. Harel. First-Order Dynamic Logic, Lecture Notes in Computer Science, 68, Springer Verlag, New York, 1979.

[5] P. Jipsen. From Semirings to Residuated Kleene Lattices, Studia Logica, 76(2), 291-303, 2004.

[6] M. Kracht. Tools and Techniques in Modal Logic, Number 142 in Studies in Logic. Elsevier, Amsterdam, 1999.

[7] D. Kozen. A representation theorem for models of *-free pdl, Report RC7864, IBM Research Center, Yorktown Heights, New York, 1979.

[8] S. Marques Pinto, M. Teresa Oliveira-Martins and M. Céu Pinto. Monadic Dynamic Algebras, Math. Log. Quart., 52(no.2), 134-150, 2006.

[9] V.R. Pratt. Dynamic Algebras: Examples, Constructions, Applications, Technical Report MIT/Laboratory for Comp. Sci/TM-138 July 1979, pp. 1-33, 1979 and Stud. Log. 50 3/4, 571-605, 1992.

[10] H. Sankappanavar and S. Burris. A Course in Universal Algebra, Springer-Verlag, New York, Heidelberg, Berlin, 1981.

[11] W. Wechler. Universal Algebra for Computer Scientists, Springer, Berlin, 1992.

[12] M. Weese. Decidable extensions of the theory of Boolean algebras, Handbook of Boolean Algebras, Volume 3, Elsevier Science Publishers, 1989.

Received 5 December 2005. Revised 24 February 2006 